\title{
Existence of a Glassy Layer in the Polymer-Nanosheet Interface: Evidence from Molecular Dynamics
}

\author{
Yangyang Gao, Jun Liu, Liqun Zhang, 'Dapeng Cao*
}

The interfacial polymer-nanosheet behavior is investigated to fully understand the interfacial interaction mechanism. The emphasis is placed on the discussion whether a "polymer glassy layer" in the proximity of the nanosheet exists or not. Results indicate that a "polymer glassy layer" actually exists near the nanosheet surface for strongly attractive interfacial interaction. Moreover, the glass transition temperatures at different interfacial interactions are explored, and a gradient of polymer chain dynamics near the nanosheet surface is observed. In short, this work provides a valuable reference for understanding polymernanosheet interfacial behavior.

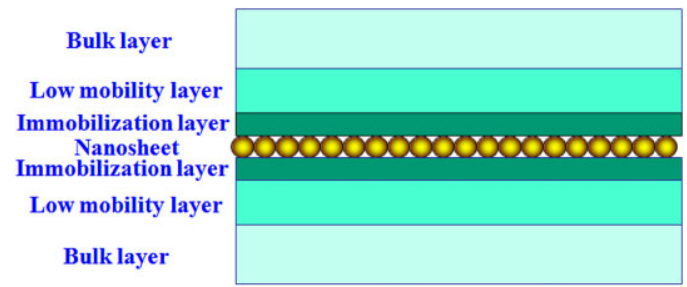

\section{Introduction}

With the development of organic-inorganic hybrid materials, such as graphite, graphene, and graphene oxide filled polymer nanocomposites, having a fundamental understanding of polymer-nanosheet interfacial behavior has become a central issue. ${ }^{[1-3]}$ Polymer chains close to a solid surface often exhibit different characteristics in their behavior compared to polymer in its bulk phase. For example, previous experimental results have indicated that the rate of the structural relaxation of glassy-state poly(methyl methacrylate) near a silica substrate surface was arrested completely, ${ }^{[4]}$ whereas for polymer/silicate intercalated nanocomposites, a

Y. Gao, J. Liu, ${ }^{[+]}$Prof. L. Zhang

Key Laboratory of Beijing City on Preparation and Processing of Novel Polymer Materials, Beijing University of Chemical

Technology, Beijing 100029, People's Republic of China

E-mail: zhanglq@mail.buct.edu.cn

Y. Gao, J. Liu, ${ }^{[+]}$Prof. L. Zhang, Prof. D. Cao

State Key Laboratory of Organic-Inorganic Composites, Beijing

University of Chemical Technology, Beijing 100029, People's

Republic of China

E-mail: caodp@mail.buct.edu.cn

${ }^{[+]}$Present address: Department of Chemical Engineering, University of Michigan, Ann Arbor, MI 48109, USA much faster new relaxation mode was observed compared to the $\alpha$-relaxation of its bulk state. ${ }^{[5]}$

In fact, extensive experimental work has been carried out to investigate the interfacial phenomenon, but, to date, no consistent conclusions have been drawn. For instance, Kaufman et al. ${ }^{[6]}$ employed nuclear magnetic resonance (NMR) and found that at least two distinct relaxation regions appeared for bound rubber, i.e., an immobile region and a relatively free region, which was also supported by results on the interfacial interactions between carbon black and cis-polybutadiene through NMR. ${ }^{[7]}$ Vo et al. ${ }^{[8]}$ employed dielectric spectroscopy to measure polymer relaxation in styrene-butadiene rubber composites, and found that a slower relaxation occurred for polymers in the interfacial region. Chen et al. ${ }^{[9]}$ used dynamic mechanical analysis to reveal the existence of double $\tan \delta$ peaks from the glass transition of bulk poly(vinyl alcohol) and an interfacial immobilized layer, which was also further investigated by Robertson et al. ${ }^{[10]}$ It was found that the occurrence of the two peaks stemmed from the restriction on the flow of polymer chains induced by the presence of particles. In contrast to the view that an interfacial immobilized layer exists near the particles, Roland et al. ${ }^{[11]}$ believed that the glass transition of a silica filled system was basically the same as that of the unfilled system, and pointed out that, although some stiffening of elastomeric chains may occur in the vicinity of fillers, a significant effect on the segmental 
dynamics was not seen. ${ }^{[12]}$ Moreover, they claimed that the segmental dynamics and glass transition were hardly affected by the polymer-filler interaction. ${ }^{[13]}$ Recently, in conjunction with experimental studies, computer simulation has played an increasingly important role in gaining a fundamental understanding of the physical properties of polymer nanocomposites (PNCs). For instance, Liu et al. ${ }^{[14]}$ employed a coarse-grained model to investigate the effects of polymer-spherical nanoparticle interactions on interfacial behavior, and did not find a "polymer glassy layer" around the spherical nanoparticles. Brown et al. ${ }^{[15]}$ performed an atomistic simulation with a silica nanoparticle (NP) embedded in an amorphous polymer matrix, and found a clear structuring and preferred orientation of polymer chains around the nanoparticle. The effect of nanoparticle size was also investigated by Brown et al., [16] and their results suggested that the size of nanoparticle had no influence on the interphase thickness for the range of particle sizes examined. However, the polymer conformation in the vicinity of the interface showed significant changes, and the range influenced around the NPs was up to $10-15 \AA$ for structural properties, which is twice that of the dynamic case. ${ }^{[17]}$ Results from Ndoro et al. ${ }^{[18]}$ indicated that chain structural properties show a larger change for higher grafting densities and a larger particle diameter.

Meanwhile, the surface shape has a significant influence on the dynamics of polymer chains in the interfacial region. ${ }^{[19]}$ Ghanbari et al. ${ }^{[20]}$ pointed out that the structure of polymer chains is strongly influenced by NPs. Starr et al. $^{[21]}$ found that there is a gradual change in polymer dynamics approaching the NP surface, which can be illustrated by a "many-layer" dynamics model. The existence of a gradient in the glass-transition temperature could explain the observed behavior and support the existence of an immobilized layer. ${ }^{[22]}$

Recently, Karatrantos et al. ${ }^{[23,24]}$ investigated the static properties, topological constraints, and dynamics of monodisperse polymer melts and found that the local chain structure, entanglement, and polymer dynamics are significantly affected by the interaction strength between the polymer and single walled carbon nanotubes. Toepperwein et al. ${ }^{[25,26]}$ theoretically found that the polymer mobility becomes slow because nanorods would induce additional topological constraints.

Meanwhile, by employing molecular dynamics (MD) simulation, Batistakis et al. ${ }^{[27]}$ found that a dramatic slowing down of polymer dynamics happens in the vicinity of a substrate surface for relatively thick films, while for thinner films a strong increase in mobility is seen. Johnston et al. ${ }^{[28]}$ have confirmed the fact that the dynamics of the polystyrene close to a surface are significantly slower than that in the bulk, which was also observed by Vagelis. ${ }^{[29]}$ Michael et al. ${ }^{[30]}$ observed that interfacial segments exhibit a jump diffusion mechanism. Correlated forward-backward motion is an important aspect of conformational relaxation, which leads to a strongly non-exponential distribution for the waiting times of dihedrals at low temperature. Komarov et al. ${ }^{[45]}$ applied an atomistic model to investigate the physical properties of polyimide/ $\mathrm{SiO}_{2}$ hybrid nanocomposites, and found a critical loading of $\mathrm{SiO}_{2}$ beyond which the material model exhibited ultra-low thermal expansion. Kumar et al. ${ }^{[46]}$ studied the effects of polymer chain length and plate separation on the microscopic conformations of polymer chains in a film, and found that the influence of a surface on chain conformations was restricted to only those segments confined within a range approximately twice the segment diameter from the surface, independent of chain length and plate separation. Meanwhile, Eslami et al. ${ }^{[4]}$ investigated the structure and mobility of nanoconfined polyamide- 6,6 oligomer, and found that the orientational relaxation times of $\mathrm{NH}, \mathrm{CO}$ and end-to-end vectors displayed oscillatory behavior, indicating well-defined ordered structures parallel to the surfaces. In addition, Kumar et al. ${ }^{[48]}$ investigated the static properties of polymer melts confined within two hard walls and found that polymer chains near the surface were flattened parallel to the surface, forming a nearly twodimensional structure. Borodin et al. ${ }^{[31]}$ investigated the effect of solid interfaces on the structure and dynamics of poly(ethylene oxide) (PEO), and found that both the surface structure and electrostatic interactions between PEO and $\mathrm{TiO}_{2}$ determine the nature of $\mathrm{PEO}$ relaxation at the $\mathrm{TiO}_{2}$ surface. Torres et al. ${ }^{[32]}$ found that the glass transition temperature was closely related to the thickness of supported polymer films.

Based on the above review, no consistent conclusion has been obtained about the interfacial behavior of PNCs from experiments. The intriguing issue of whether "glassy polymer layers" or completely immobilized polymer chains exist around the filler has not yet been resolved. Most previous simulation works have been focused on spherical NP-filled polymer systems. Here, polymer-nanosheet systems have been studied with the aim of exploring whether or not a "glassy polymer layer" near the nanosheet exists by tuning interfacial interaction. In fact, in experiments, the size of spherical NPs will be hundreds of times larger than one polymer segment, and the surface of the NPs approximates a flat plane compared to the size of the polymer segments. This research is therefore also applicable for experimental work on PNCs filled with spherical NPs. In this study, the microscopic structural characteristics (such as number density distribution, orientation, chain size, glass transition temperature) and the dynamic properties (interfacial adsorption/desorption dynamics, translational and orientation mobility) were explored. It should be noted that the simulated systems were built based on two preliminary conditions, i.e., the nanosheet is

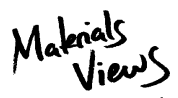

www.MaterialsViews.com

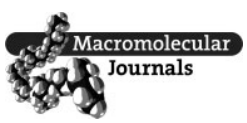


completely exfoliated and the volume fraction of the nanosheet is not very high.

\section{Models and Simulation Methods}

The idealized polymer model consists of thirty beads with its diameter equal to $\sigma .^{[33]}$ The total number of simulated polymer beads is 6900 . The single nanosheet is comprised of 400 beads $(20 \times 20)$ with its diameter equal to $\sigma$. Although these chains are rather short, they capture the basic static and dynamic features of polymer chains. We set the mass of the polymer bead equal to $m$, and the radius and mass of the nanosheet bead are the same as those of the polymer bead. Following the literature, ${ }^{[34-36]}$ we used the modified Lennard-Jones (U) interaction to model the polymerpolymer, polymer-nanosheet, and nanosheet- nanosheet interactions, given by Equation (1):

$$
U(t)=\left\{\begin{array}{c}
4 \varepsilon\left[\left(\frac{\sigma}{r}\right)^{12}-\left(\frac{\sigma}{r}\right)^{6}\right]+C \quad r<r_{\text {cutoff }} \\
0 \quad r \geq r_{\text {cutoff }}
\end{array}\right.
$$

where $C$ is a constant $\left(C=-4 \varepsilon\left[\left(\frac{\sigma}{r_{\text {cutoff }}}\right)^{12}-\left(\frac{\sigma}{r_{\text {cutoff }}}\right)^{6}\right]\right)$ satisfying that the potential energy is continuous everywhere. $r_{\text {cutoff }}$ stands for the distance at which the interaction is truncated and shifted so that the energy and force are zero. The polymer-polymer interaction and its cutoff distance are $\varepsilon_{p p}=1.0$ and $r_{\text {cutoff }}=2 \times 2^{1 / 6}$, and the nanosheet-nanosheet interaction and its cutoff distance are set to $\varepsilon_{n n}=1.0$ and $r_{\text {cutoff }}=2.5$, while the polymer-nanosheet interaction $\varepsilon_{s p}$ and the cutoff distance $r_{\text {cutoff }}$ are changed to simulate different interfacial interaction strengths and ranges. For better characterization of the interfacial behavior between the polymer and the nanosheet, the single nanosheet is immobilized. This is physically reasonable since, in a practical situation, the large sheet always exhibits small thermic Brownian motion.

Additionally, the interaction between the adjacent bonded monomers is represented by a stiff finite extensible non-linear elastic (FENE) potential, as shown in Equation (2):

$$
V_{F E N E}=-0.5 k R_{0}^{2} \ln \left[1-\left(\frac{r}{R_{0}}\right)^{2}\right]
$$

where $k=30 \frac{\varepsilon}{\sigma^{2}}$ and $R_{0}=1.5 \sigma$, guaranteeing a certain stiffness of the bonds while avoiding high-frequency modes and chain crossing.

Since it was not the aim of this work to study a specific polymer, the $\mathrm{L}$ units where $\varepsilon$ and $\sigma$ are set to unity were used. This means that all calculated quantities are dimensionless. To generate the initial configurations, the polymer chains and nanosheet were placed in a box to keep the number density of polymers at around $\rho^{*}=0.85$, which corresponds to a dense melt state. Periodic boundary conditions were employed in all three directions. The velocity-Verlet algorithm was used to integrate the equations of motion, with a time step $\delta t=0.001$, where the time is reduced by the $\mathrm{L}$ time $(\tau)$. An NVT ensemble was adopted, where the temperature is fixed at $T^{*}=1.0$ using the Nose-Hoover temperature thermostat. All structures were equilibrated over a long time so that each chain had moved at least $2 R_{g}\left(R_{g}\right.$ is the root-mean-squared radius of gyration). For strong interaction $\left(\varepsilon_{s p}>2\right)$, the equilibrium configuration of low interaction was used as the starting point, only tuning $\varepsilon_{s p}$. All MD runs were carried out using the large scale atomic/molecular massively parallel simulator (LAMMPS) developed by Sandia National Laboratories. ${ }^{[37]}$

\section{Results and Discussion}

\subsection{Density Profiles}

Here three different kinds of polymer-filler interactions were chosen: (i) $\varepsilon_{s p}=1.0$ and $r_{\text {cutoff }}=1.12$; (ii) $\varepsilon_{s p}=1.0$ and $r_{\text {cutoff }}=2.5$; (iii) $\varepsilon_{s p}=10.0$ and $r_{\text {cutoff }}=2.5$. These represented purely repulsive, weakly, and strongly attractive polymernanosheet interactions, respectively. Note that when mapping this bead-spring model to a real polymer, each bond corresponds to 3-6 covalent bonds along the backbone of a real chain, making the bead diameter $\sigma$ approximately range from 0.5 to $1 \mathrm{~nm}$. Meanwhile, the interaction parameter $\varepsilon$, representing the van der Waals interaction between two polymer segments, varied from 2.5 to $4 \mathrm{~kJ} \mathrm{~mol}^{-1}$ for different polymers. To clearly characterize the adsorption state of polymer chains on the nanosheet surface, snapshots are shown in Figure 1(a) for three different polymer-nanosheet interactions. The polymer chain is away from the nanosheet surface for a purely repulsive interaction, while it is adsorbed on the attractive surface, indicating a wetting behavior. The density profiles of the systems for three different polymer-nanosheet interactions are shown in Figure 1 (b), where $Z<10.5$ stands for one side and $Z>10.5$ for the other side of the nanosheet. For example, $Z=8.5 \sigma$ meant that the positions of the polymer beads were a distance (10.5$8.5=2 \sigma)$ from the nanosheet.

Figure 1 (b) shows that the polymer chains are distributed in five interfacial layers, denoted as L1, L2, L3, L4, and L5. In particular for L1, the bead density near the nanosheet surface greatly exceeds the bulk density for strongly attractive interaction. Bead layering close to the nanosheet strongly depends on the polymer-nanosheet interaction. For the repulsive case, the observed ordering is an entropic effect, while for the full $L$ potential $\left(r_{\text {cutoff }}=2.5\right)$, the 
(a)

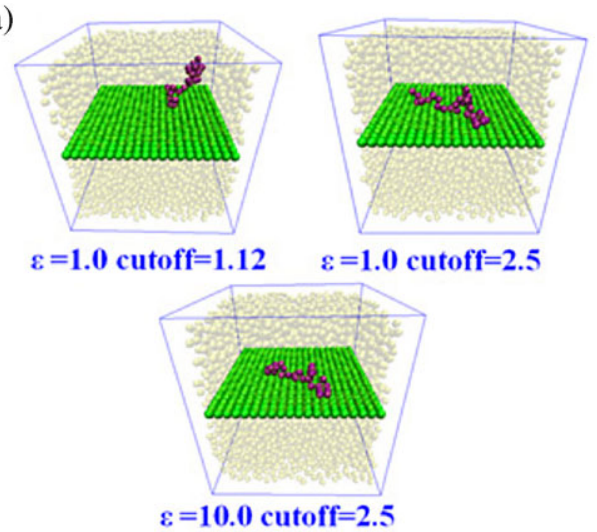

(b)

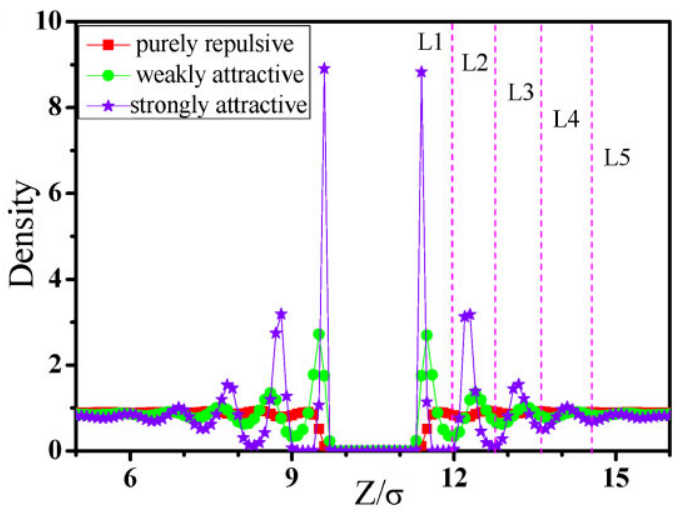

(c)

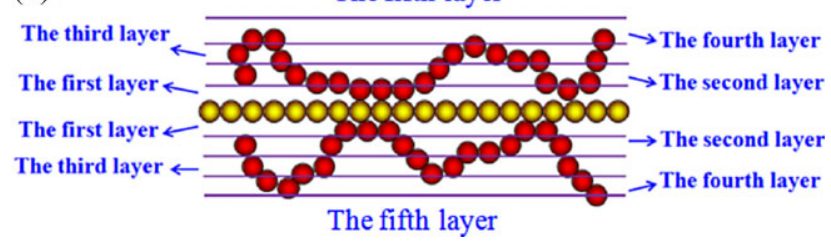

Figure 1. (a) Snapshots for three different polymer-nanosheet interactions, where the dark yellow spheres denote polymer beads, the green spheres denote the nanosheet and the purple spheres represent one single polymer chain located in the vicinity of the nanosheet. (b) Density profiles for the three different polymer-nanosheet interactions, where the vertical axis at $Z=10.5 \sigma$ denotes the position of the nanosheet surface. $L_{1}, L_{2}$, $\mathrm{L}_{3}, \mathrm{~L} 4$, and $\mathrm{L}_{5}$ denote five interfacial layers. (c) Schematic illustrations of the position distribution of two polymer chains in the proximity of the nanosheet surface, where different layers are defined according to the density profiles.

layering is enhanced. The positions of the two polymer chains in the proximity of the sheet surface are displayed in Figure 1(c).

\subsection{Ordering of Polymer Chains}

The structure of the polymer chains near the filler was characterized at the bond, segment (five beads), and chain length scales by the second-order Legendre polynomials
$<P_{2}(\cos \theta)>$, given by Equation (3):

$$
\left\langle P_{2}(\cos \theta)\right\rangle=\frac{3}{2}\left\langle\cos ^{2} \theta\right\rangle-\frac{1}{2}
$$

At the bond length scale, $\theta$ denotes the angle between the bond vector and the normal direction of the nanosheet surface. At the segment length scale, those segments connecting beads $i$ and $i+4$ in a polymer chain are conventionally assigned to the bin, in which the middle point of the vector locates. At the chain length scale, $\theta$ stands for the angle between the end-to-end vector and the normal direction of the nanosheet surface. $<P_{2}(\cos \theta)$ is zero if the bond, segment or chain vectors are randomly oriented. If the bond, segment or chain vectors are parallel to the nanosheet surface, $P_{2}(\cos \theta)$ is equal to $-1 / 2$. Therefore, any bond, segment or chain vector with its preferential orientation tangential to the nanosheet surface will result in a negative $P_{2}(\cos \theta)$ between $-1 / 2$ and 0 .

The simulated bond, segment, chain orientations and bond length are shown in Figure 2. In the vicinity of the nanosheet surface (Figure 2(a)), the oscillation in bond orientation is very large, and it gradually diminishes as it moves away from the nanosheet surface. The $P_{2}(\cos \theta)$ approaches -0.5 near the nanosheet surface, indicating the bond orientation is parallel to the surface. Several peaks appear as it moves away from the surface. The peak height and position depends on the polymer-nanosheet interaction. Interestingly, the change in $P_{2}$ coincides well with the change in the density profile in Figure $1(b)$, i.e., the higher the atom density along the Z-axis, the lower the order parameter. Thus, the valleys of the order parameter correspond to the peaks of the density profiles and vice versa, which means that the polymer beads within the layers are parallel to the sheet surface, while those located between layers are perpendicular to the surface. However, the segment and chain orientations become a little different. Since the segment and chain scales are larger than the bond scale, the oscillation in segment orientation is smaller than that in the bond orientation (Figure 2(b) and 2(c)). The segment and chain order parameters approach -0.5 near the nanosheet surface, then increase to zero as it moves away from the nanosheet surface for two low interfacial strengths. These results basically agree well with the literature. ${ }^{[38,39]}$ Apparently, the bond is elongated or shortened in the vicinity of the nanosheet surface (see Figure 2(d)), which is induced by large polymer-nanosheet interaction energies. The bond length is around $1.01 \sigma$ near the nanosheet surface, then decreases to a valley. The depth and position of this valley depend on the polymernanosheet interaction. Clearly, the variation trend in the bond length is similar to the bond orientation behavior. When the distance between the center of the bond, segment, chain and nanosheet surface is beyond $5 \sigma$, the value of $P_{2}(\cos \theta)$ is close to zero, suggesting that they are in a

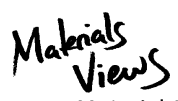

www.MaterialsViews.com 

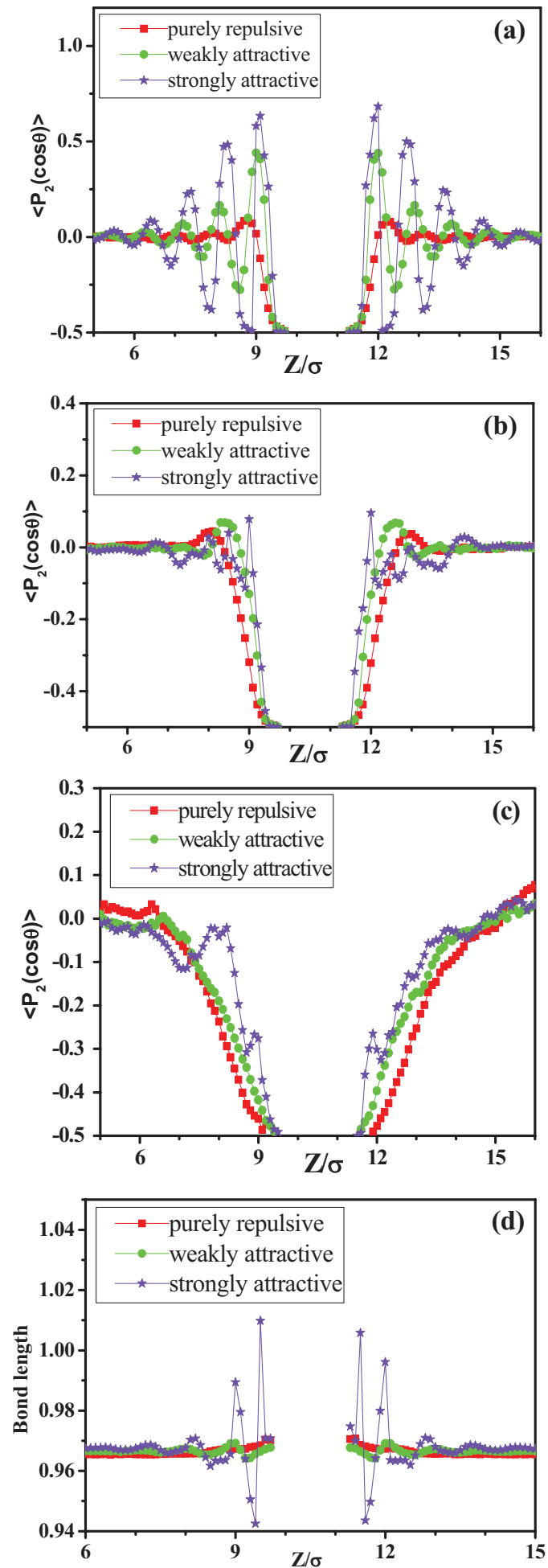

Figure 2. The second-order Legendre polynomials $P_{2}(\cos \theta)$ (a) for bond orientation, (b) for segment orientation, (c) for chain orientation, and (d) bond length as a function of the distance from the surface of the nanosheet. random bulk state. The interface width is therefore around $5 \sigma$ in our systems. Based on these results, the orientations of the bond, segment and chain in the direction tangential to the nanosheet surface are induced by the interfacial enthalpy and the filler geometric constraints.

\subsection{The Mean Square Radius of Gyration and End-to-End Distance}

The conformation of the polymer chains was examined by calculating the mean square radius of gyration $R_{g}^{2}$ and endto-end distance $R_{\text {ete }}^{2}$, as shown in Figure 3. Obviously, for all three different polymer-nanosheet interactions (see Figure 3(a) and 3(b)), an appreciable increase of $R_{g}^{2}$ and $R_{\text {ete }}^{2}$ is seen in the vicinity of the nanosheet surface, indicating that the chain dimension is enlarged, which is independent of the polymer-nanosheet interaction. It is well known that a good indicator of geometrical conformation for a polymer chain is the gyration tensor $Q$, given by Equation (4):

$$
\underline{Q}_{\alpha \beta}=\frac{1}{N} \sum_{j=1}^{N}\left(R_{j, a}-R_{c m, \alpha}\right)\left(R_{j, \beta}-R_{c m, \beta}\right)(\alpha, \beta=1, \ldots 3)
$$

Here, $R_{j, \alpha}$ and $R_{c m, \alpha}$ denote the $\alpha$-th component of the three-dimensional position vectors to the $\mathrm{j}$-th monomer and to the mass center of the chain, i.e., $R_{j}=\left(R_{j, 1}, R_{j, 2}, R_{j, 3}\right)$ and $R_{c m}=\left(R_{c m, 1}, R_{c m, 2}, R_{c m, 3}\right)$. The three average eigenvalues $\left(\lambda_{1}, \lambda_{2}, \lambda_{3}\right)$ of $Q$ sum up to the mean square radius of gyration in Equation (5):

$$
\lambda_{1}+\lambda_{2}+\lambda_{3}=R_{g}^{2}
$$

The three components $\lambda_{1}, \lambda_{2}$, and $\lambda_{3}$ were analyzed as a function of the distance from the surface, and are shown in Figure $3(\mathrm{c})$. The results are symmetrized along the $\mathrm{z}$-axis and the two in-plane components are averaged. In all cases of the in-plane components, $\lambda_{p}=\frac{1}{2}\left(\lambda_{x x}+\lambda_{y y}\right)$ are larger at the surface whereas the $z$ components are smaller. Therefore, the conformations are compressed along the z-direction and elongated along the surface, which means that the chains are lying along the surface. Interestingly, the altered region in Figure 3 (c) is also in the range of distance of around $5 \sigma$, beyond which the chains exhibit random bulk behavior. Moreover, the changes in chain size and shape are independent of the polymer-nanosheet interaction, suggesting that the effects completely originate from geometric constraints. Here the behavior of the chain size and shape within the space around $5 \sigma$ from the nanosheet surface could be ascribed to the configurational entropy. 

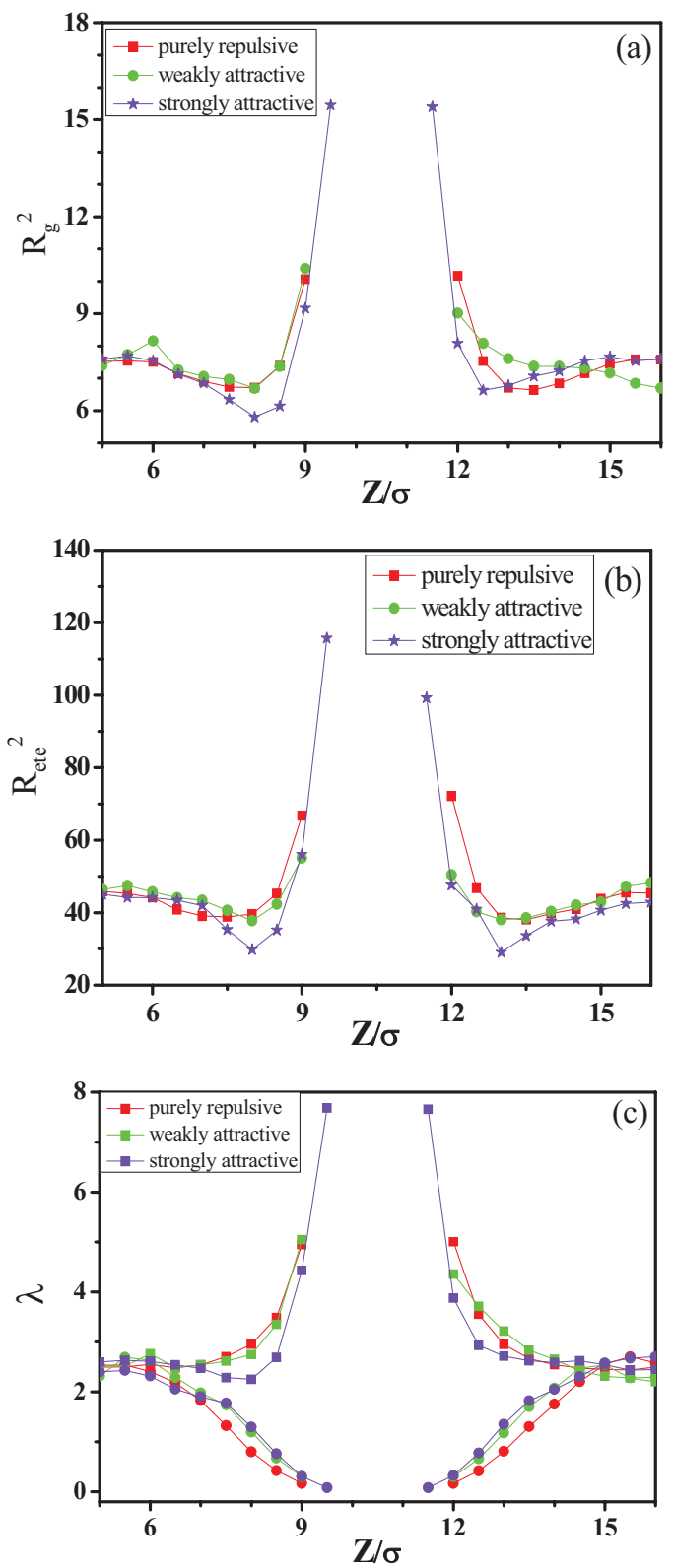

Figure 3. Change of (a) the mean squared radius of gyration and (b) the mean squared end-to-end distance with respect to the distance between the chain center of mass and the surface of the nanosheet. (c) Variation of eigenvalues of the gyration tensor of chains for different polymer-nanosheet interactions. Squares show the components averaged in the surface (xy) plane and circles represent the component perpendicular to the surface.

\subsection{Glass Transition Temperature}

The glass-transition temperature $\left(T_{g}\right)$ can be identified using the temperature dependence on the volume when it is cooled or heated under a constant external pressure. The box dimensions in the xy-plane were fixed, and the pressure was only applied along the $z$-axis. The $T_{g}$ for pure polymer was also simulated under the same external pressure, and

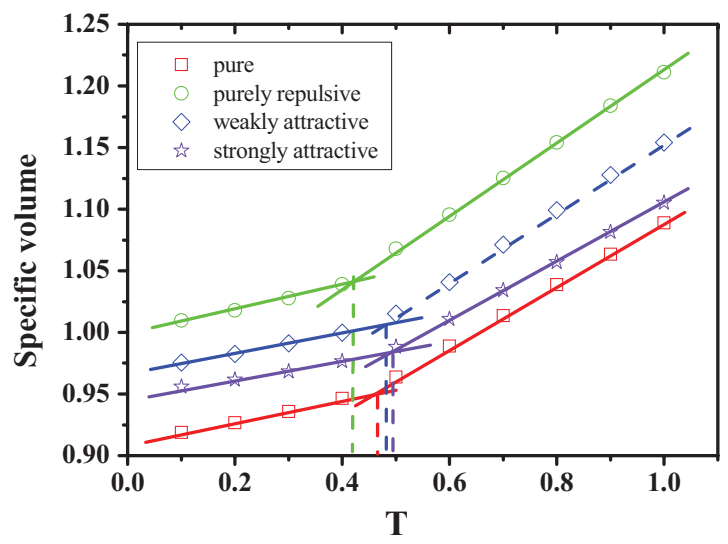

Figure 4. Plot of the specific volume of the neat polymer as well as for samples with purely repulsive, weakly and strongly attractive polymer-nanosheet interactions as a function of temperature. Note that the simulated pressure is fixed at $P=1.0$.

in this case, the pressure was applied in all three directions. The specific volume for four different systems is plotted in Figure 4, where the glass transition is marked at the intersection of the linear fits. Since the cooling or heating rate could influence the glass transition temperature to some extent, the cooling rate was fixed in these simulations for consistency, and it took $2000 \tau$ to decrease the temperature by $0.1 T^{*}$. In general, $T_{g}$ slightly increased for the attractive polymer-nanosheet interaction, compared with the pure system, and $T_{g}$ of the repulsive system was slightly smaller than that of pure system. This observation is consistent with experimental results where the increas $\mathrm{e}^{[40]}$ or decrease ${ }^{[41]}$ of $T_{g}$ depends on the strength of the polymer-filler interaction.

\subsection{Dynamical Properties}

\subsubsection{MSD of Polymer Beads}

Next, the chain dynamics in the vicinity of the nanosheet were studied for three systems. To explore the mobility of the polymer chains, the mean-square displacement (MSD) in a short time of $50 \tau$ was calculated. For better comparison, the MSD of the polymer chains as a function of temperature in the pure system is plotted in Figure 5(a), where the temperature at the intersection point of two lines is 0.47 , corresponding to the glass transition temperature and the mobility of polymer chains in the glassy state becoming nearly frozen. Furthermore, the MSD was monitored for three different interfacial interactions, as shown in Figure $5(b)$. The chain mobility decreased in the vicinity of the nanosheet for the attractive cases, and was almost frozen for the strongly attractive case, while the chain mobility increased for the purely repulsive situation. This indicates that a "glassy polymer layer" may exist near the 

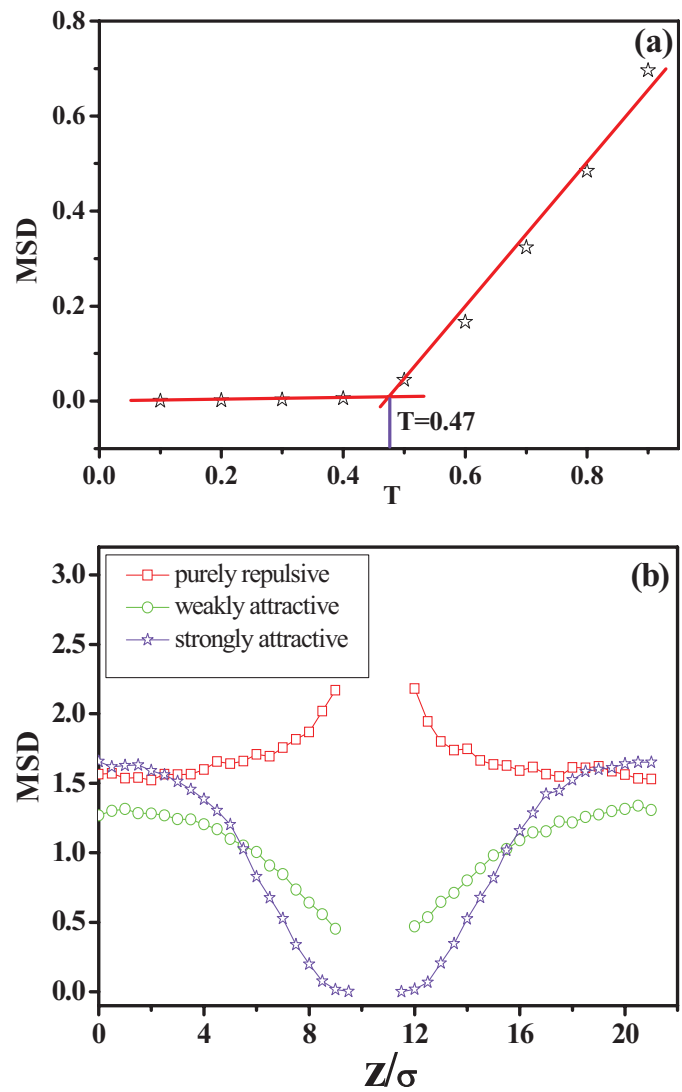

Figure 5. (a) Mean-square displacement (MSD) of the polymer chains as a function of temperature for a pure system. The intersection point of the two lines corresponds to a temperature equal to 0.47. (b) MSD of the polymer chains as a function of the distance from the surface of the nanosheet for different polymer-nanosheet interactions. The MSD is calculated for a short time of $50 \tau$.

nanosheet surface. Meanwhile, it was also observed that the behavior of the bulk mobility recovered beyond $5 \sigma$. It was noted that the MSD of the bulk polymer chains was a little different for the three systems, which could be a result of different bead densities.

\subsubsection{Spatial Distribution of Polymer Beads in the Two} Layers

Figure 6 and Figure 7 show the spatial distribution and corresponding concentration profiles of the population of beads located in the first two layers close to the surface. After selecting the chains that are exactly located in the first two layers close to the surface, this time is defined as the initial time. Then chains continue to move and their behavior is monitored. The adsorption snapshots for weakly and strongly attractive interfacial interactions at time (1) $0 \tau$, (2) $500 \tau$, (3) $3000 \tau$ and (4) $9000 \tau$ are displayed in Figure $6(\mathrm{a})$ and $7(\mathrm{a})$. Obviously, the desorption kinetics are (a)

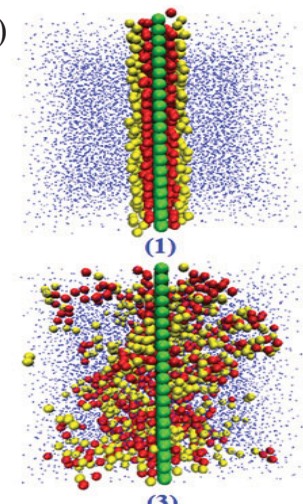

(3)

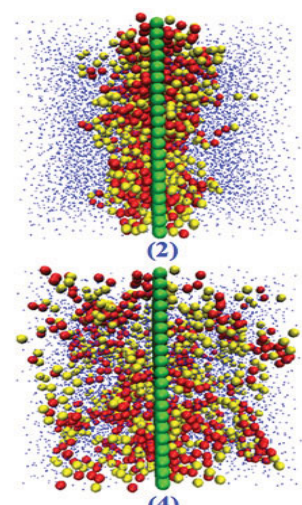

(4)

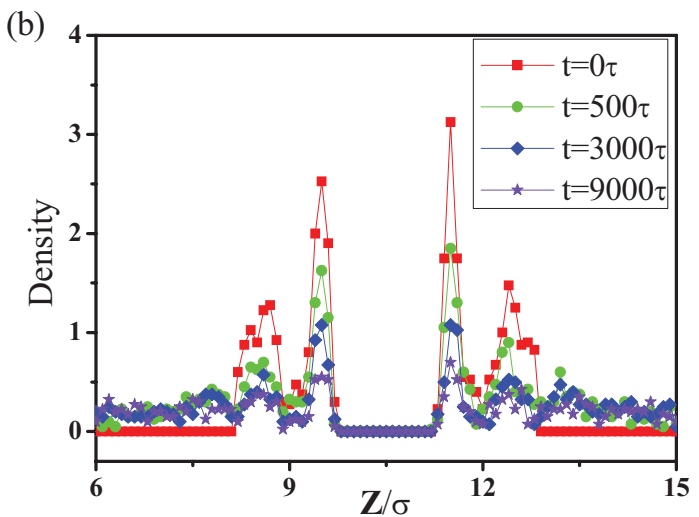

Figure 6. (a) Snapshots of the distribution of polymer beads at time (1) $0 \tau$, (2) $500 \tau$, (3) $3000 \tau$, and (4) $9000 \tau$ for weakly attractive interfacial interaction. The red spheres denote polymer beads adsorbed in the first layer, the yellow spheres denote polymer beads located in the second layer, the blue points represent the polymer beads outside the two layers at the initial time, and the green spheres model the nanosheet. (b) The change in the corresponding concentration profiles as a function of time.

faster for the weakly attractive interaction. The number density of beads declines with time (see Figure $6(b))$. For strongly attractive interfacial interaction, it is very difficult for the beads in the second layer to diffuse into the bulk, and the beads in the first layer basically stay in their original positions during the whole simulation (see also Figure 7(a)). Correspondingly, the density of beads near the surface almost does not change, as shown in Figure $7(b)$, which suggests the formation of a "glassy bead layer".

To more clearly examine the interfacial chain mobility, a polymer chain with some beads adsorbed in the first layer at the initial time was randomly selected. This chosen polymer chain is a typical representative of the motion of other chains in the first layer. Then, the motion of thirty beads of this polymer chain was traced by calculating the distance between every bead and nanosheet as a function of time. The results for the two attractive systems are shown in Figure 8 and Figure 9. In Figure $8(\mathrm{a})$, at time $t=0$ the beads of this polymer chain are located in different layers, 
(a)
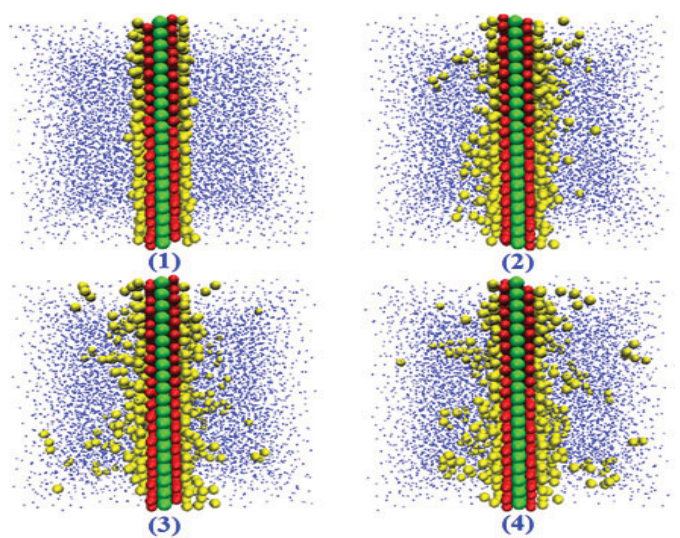

(b)

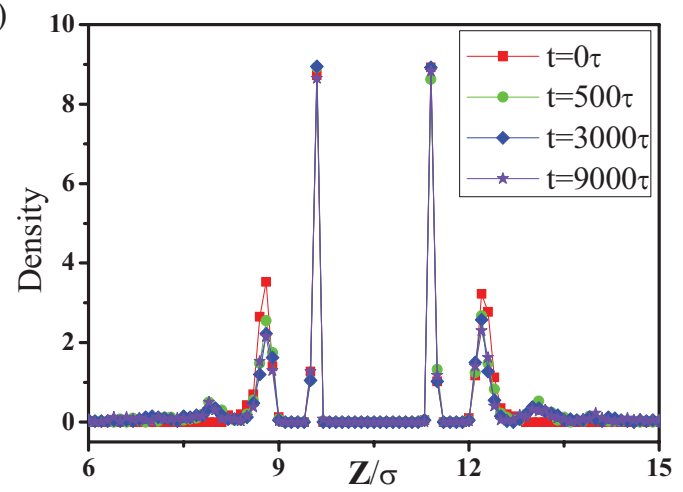

Figure 7. (a) Snapshots of the distribution of polymer beads at time (1) $0 \tau$, (2) $500 \tau$, (3) $3000 \tau$, and (4) $9000 \tau$ for strongly attractive interfacial interaction. The red spheres denote polymer beads adsorbed in the first layer, the yellow spheres denote polymer beads located in the second layer, the blue points represent the polymer beads outside the two layers at the initial time, and the green spheres model the nanosheet. (b) The corresponding concentration profiles.

including the first and second layers. At time $t=1000 \tau$, those beads in the first layer at $t=0 \tau$ move to other layers (denoted as circles), and some beads in other layers at $t=0 \tau$ move to the first layer. A similar situation is also observed at time $t=2000 \tau$, but all beads left the first layer. However the situation is completely different for strongly attractive interaction, as shown in Figure 9(a). The beads initially located in the first layer nearly do not move, even at $t=10000 \tau$. The beads outside the first layer move slightly, but their motility is also inhibited by these "glassy beads", compared to the case of weakly attractive interaction. Furthermore, the distance between the chain center of mass and the nanosheet is plotted in Figure 8(b) and Figure $9(b)$. Similarly, it is found that the center-center distance for the weakly attractive system shows an increase trend and a big fluctuation, while the center-center distance for the strongly attractive system almost does not change. Meanwhile, Figure 9(c) shows the mean square displacement (MSD) of interfacial chains and glassy chains for the
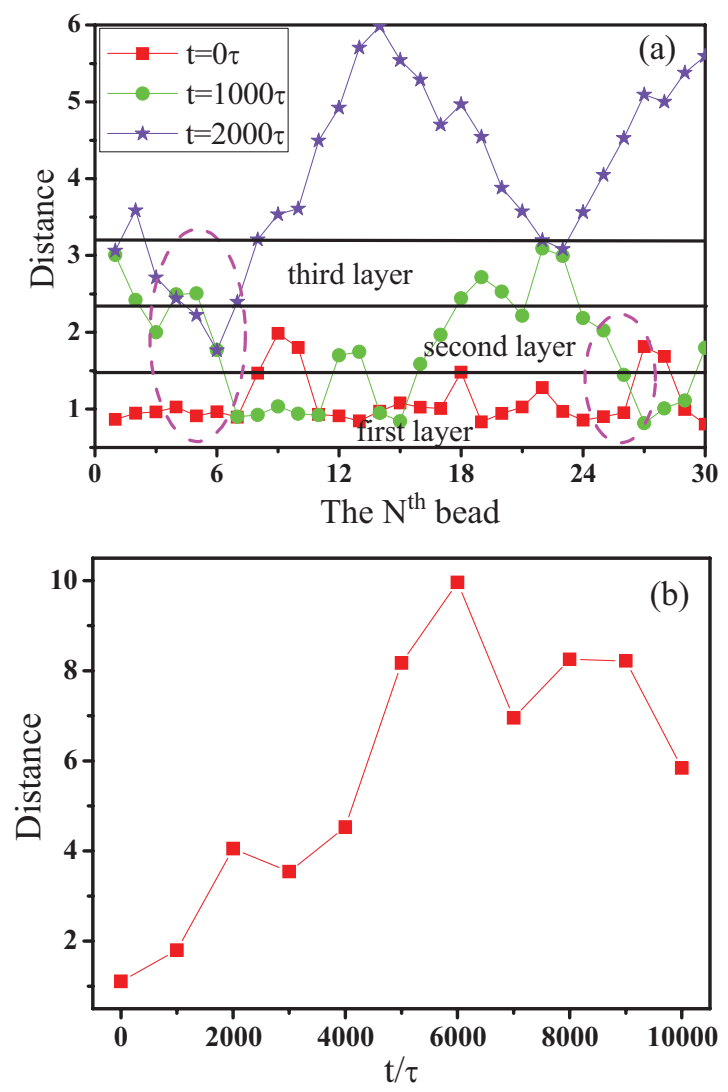

Figure 8. (a) The trajectory of one polymer chain initially adsorbed in the vicinity of the nanosheet for weakly attractive interaction. The broken oval rings denote the typical zones where polymer beads can move between different layers. (b) The change of the distance between the mass center of the chosen polymer chain and the surface of the nanosheet for weakly attractive interaction.

strongly attractive system. The distance of the chain and nanosheet is defined through its center of mass, so it is possible to determine which layer the polymer chain belongs to. It was found that the polymer chains in the vicinity of the nanosheet $(d<1.0 \sigma)$ are immobilized completely, suggesting the formation of a "glassy polymer layer". The interfacial polymer chains $(d<1.0 \sigma)$ completely cannot undergo the adsorption/desorption process, which is attributed to nanoscale confinement and interfacial interactions. These studies indicate the following points: (1) In the interfacial region, some beads of polymer chains are adsorbed on the nanosheet surface (in the first layer) and other beads can span over other layers. (2) The polymer chains adsorbed on the surface can be desorbed from the surface for a weakly attractive system and exchanged with those polymer chains outside the layer $(d>1.0 \sigma)$, but the polymer chains $(d<1.0 \sigma)$ almost do not move for a strongly attractive system with the evolution of time. (3) When the distance of the polymer chain and the nanosheet is less than

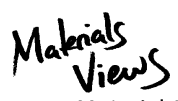

www.MaterialsViews.com 

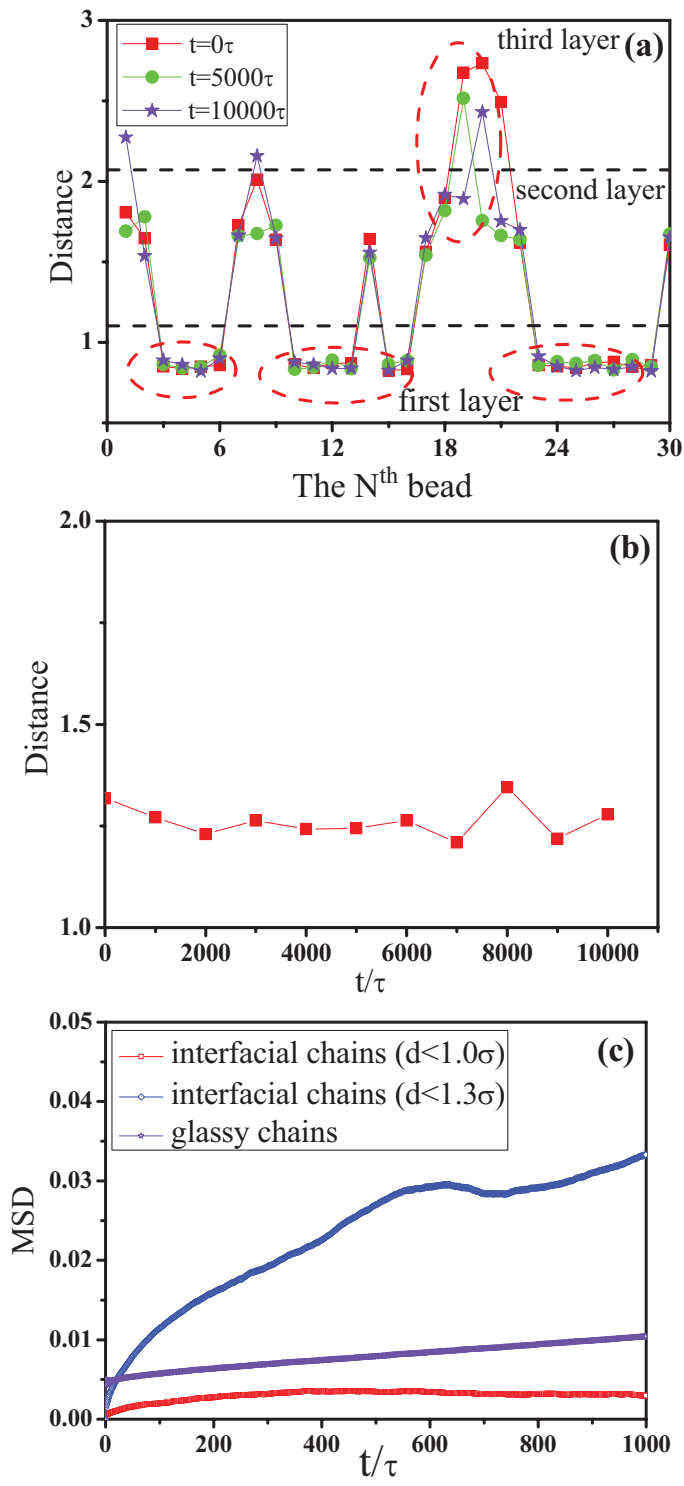

Figure 9. (a) The trajectory of one polymer chain initially adsorbed in the vicinity of the nanosheet for strongly attractive system. The broken oval rings denote the typical zones where polymer beads only stay in the initial position. (b) The change of the distance between the mass center of the polymer chain in (a) and the surface of the nanosheet. (c) Mean square displacement (MSD) averaging for chains in the interfacial region, namely the distance between the mass center of the polymer chains and the nanosheet is smaller than $d=1.3 \sigma$ and $d=1.0 \sigma$. For comparison, the MSD of polymer chains in the glassy state $\left(T<T_{g}\right)$ is also shown. For example, for $d<1.3 \sigma$, chains are only counted when the distance between the center of chain mass and the nanosheet surface is less than $1.3 \sigma$.

$1.3 \sigma$ (i.e., $d<1.3 \sigma$ ), a "glassy bead layer" is formed and the beads within the contact layer are basically frozen, while when $d<1.0 \sigma$, a "glassy polymer layer" is formed and the polymer chains within the contact layer are completely frozen. Obviously, the "glassy polymer layer" is

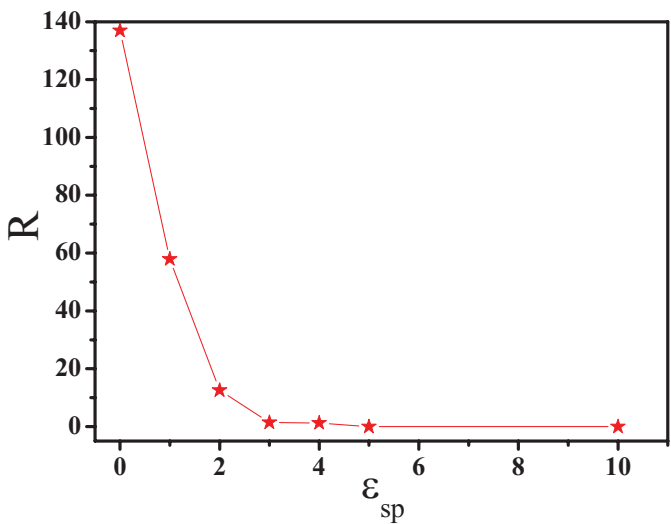

Figure 10. The average number of beads undergoing adsorption/ desorption process as a function of polymernanosheet interfacial interaction.

characterized on the segment or chain length scales, while the "glassy bead layer" is referred to on the bead length scale. Accordingly, our research indicates that a "glassy polymer layer" can be formed near the nanosheet for strongly attractive interaction.

\subsubsection{Adsorption/Desorption Rate}

It is obvious that polymer beads can subsequently be adsorbed on the surface, and then be desorbed if the "glassy layer" does not exist. So, we examined the exchange rate $(R)$ of polymer beads on the nanosheet surface, which is defined as the cumulative number of adsorption and desorption events. As seen in Figure 10, the exchange rate shows a dramatic decrease from 137 to 0 with the increase of polymer-nanosheet interaction. The exchange rate is rather high for low interfacial interaction strength, while for the highest interfacial strength, the exchange rate is 0 , which means that the interfacial beads cannot move into the bulk and the exchange dynamics cease completely. Accordingly, we can infer that the "glassy bead layer" also exists near the nanosheet for the strongest attractive interaction.

\subsubsection{Orientation Mobility and Desorption Dynamics}

To gain an insight into the effect of the nanosheet surface on the polymer dynamical behavior, the local orientation mobility of polymers located in different layers is characterized through the autocorrelation function (ACF) in Equation (6):

$$
P_{1}(t)=\langle\cos (\theta(t))\rangle
$$

where $\theta(t)$ is the angle between the bond vector at time $t$ and the vector at time $t=0$, and the bond vector refers to the vector between two connected beads in the polymer chains. The dynamics of the polymer chains were studied at the 
bond scale defined above. The layer to which the bond belonged was defined according to the initial position of the mass center of the bond.

The effect of the polymer-nanosheet interfacial interaction on the bond orientation dynamics was analyzed and is shown in Figure 11(a) and 11(b) for the purely repulsive and strongly attractive cases, respectively. For the purely repulsive system, the bond mobility dynamics in the first layer increase slightly compared to its bulk state. However, for the strongly attractive systems, the bond mobility gradually became slower when approaching the nanosheet surface. Polymer beads in the first layer almost did not move for the strongly attractive case. As a result, the value of $P_{1}(t)$ is nearly equal to 1 , indicating that the polymer beads adsorbed in the first layer are immobilized completely.

To obtain quantitative information on the relaxation time from the ACF, the data was fitted with the stretched exponential Kohlrausch-Williams-Watts (KWW) function, given by Equation (7):

$$
P_{1}(t)=\exp \left(-\left(\frac{t}{t_{K W W}}\right)^{\beta}\right)
$$

where $t_{K W W}$ is the relaxation time and $\beta$ is the stretch exponent. However, the R-square is less than 0.99 for the bond orientation mobility. The reason is that a bond can jump outside the layer or can fluctuate between two layers during the simulation, which affects the bond dynamics in each layer. To obtain a parameter that can characterize the bond orientation mobility, the average lifetime was measured (Equation (8)):

$$
\tau_{\text {avg }}=\int_{0}^{\infty} P_{1}(t) d t
$$

To further quantify the effect of the interface on the dynamics of the polymer beads, the $\tau_{\text {avg }}$ for bond orientation mobility was extracted and is shown in Figure 11(c). The beads in the first layer of the purely repulsive interaction exhibit a rapid relaxation compared to its bulk value, while the relaxation dynamics in the first layer show a dramatic decrease for the attractive interaction (see Figure 11(c)). At the same time, the plateau value became large when approaching the nanosheet surface (see Figure 11b).

Then, the intrinsic dynamics of the population of the interfacial polymer beads were explored. This population is continually exchanging beads with the rest of the polymer melt. To characterize the dynamics, the correlation functions were calculated using Equation (9):

$$
G_{\text {bead }}(t)=\left\langle n_{\text {first-layer }}(t) / n_{\text {first-layer }}(0)\right\rangle
$$
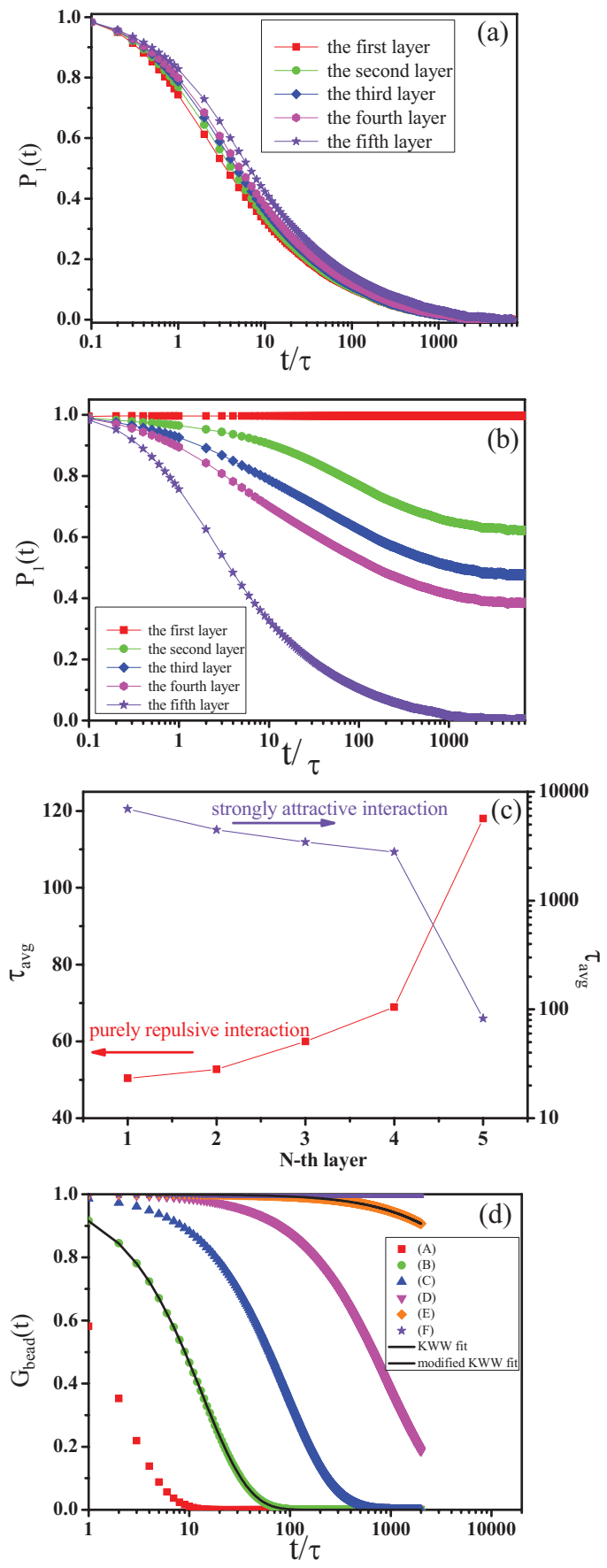

Figure 11. The decay of the bond-orientation function $P_{1}(t)$ for different layers parallel to the nanosheet surface, for temperature $T=1$ : (a) for the purely repulsive system; (b) for the strongly attractive system. (c) The average lifetime $\tau_{\text {avg }}$ for the bond orientation mobility in different adsorption layers for purely repulsive and strongly attractive interfacial interaction strength. (d) Decay of $G_{\text {bead }}(t)$ for different polymer-nanosheet interfacial strengths: (A) $\varepsilon_{s p}=1.0, r_{\text {cutoff }}=1.12$; (B) $\varepsilon_{s p}=1.0, r_{\text {cutoff }}=2.5$; (C) $\varepsilon_{s p}=2.0, \quad r_{\text {cutoff }}=2.5 ; \quad$ (D) $\varepsilon_{s p}=3.0, \quad r_{\text {cutoff }}=2.5 ; \quad(\mathrm{E}) \varepsilon_{s p}=4.0$, $r_{\text {cutoff }}=2.5$; F) $\varepsilon_{s p}=10.0, r_{\text {cutoff }}=2.5$. The line represents the KWW fit for (B) and the modified KWW fit for (E), respectively. Fits for other cases are not shown for clarity. 
in which $n_{\text {first-layer }}(t)$ is the number of polymer beads in the first layer at time $t$. In particular, the evolution of beads in the first layer at $t=0$ is interesting. At the initial time $(t=0)$, those beads located in the first layer are labeled, and, after time $t$, some labeled beads left the first layer. The number of labeled beads that remained in the first layer were counted. Thus, $G_{\text {bead }}(t)$ is a measure of the fraction which never left the surface over a period of time $t$, which describes only the desorption kinetics. For accurate comparisons, the size of the interval must be the same for all systems, and the interval was chosen to be $1 \tau$.

The effect of the polymer-nanosheet interfacial interaction on the bead desorption dynamics is shown in Figure 11 (d). For weak interfacial interaction, the beads can be desorbed from the nanosheet surface, while with an increase in the interfacial interaction, the desorption dynamics show a dramatic decrease. Polymer beads in the first layer almost do not move for the strongest attractive case, resulting in a value of $G(t)$ nearly equal to 1 , which reconfirms that the polymer beads adsorbed in the first layer are fully immobilized.

For weak interfacial interaction, the KWW function fitted these curves well, as shown in Figure 11(d) for weak interfacial strength. However, for the strong interfacial strength with the occurrence of a plateau, the modified KWW function was used to fit the decay of $P_{1}(t)$, given by Equation (10):

$$
P_{1}(t)=(1-\alpha)^{*} \exp \left(-\left(\frac{t}{t_{K W W}}\right)^{\beta}\right)+\alpha
$$

Impressively, this modified KWW function fitted the curves very well, as shown in Figure 11(d). For a better understanding, (A)-(F) were used to represent the following six different systems. The chosen parameters were $(A)$ $\varepsilon_{s p}=1.0, r_{\text {cutoff }}=1.12$; (B) $\varepsilon_{s p}=1.0, r_{\text {cutoff }}=2.5$; (C) $\varepsilon_{s p}=2.0$, $r_{\text {cutoff }}=2.5 ;$ (D) $\varepsilon_{s p}=3.0, r_{\text {cutoff }}=2.5$; (E) $\varepsilon_{s p}=4.0, r_{\text {cutoff }}=2.5$; (F) $\varepsilon_{s p}=10.0, r_{\text {cutoff }}=2.5$. For desorption of beads, the relaxation $t_{K W W}$ are 1.921 for $\mathrm{A}, 13.38$ for $\mathrm{B}, 95.07$ for $\mathrm{C}$, 897.5 for $D$, 5140 for $E$ and $\infty$ for $F$ and the plateau value $\alpha$ is 0 for A-C, 0.0637 for D, 0.7271 for $E$ and 1 for F. They exhibit a dramatic increase with increasing the polymer-nanosheet interfacial strength. In particular, the polymer beads in the first layer are completely frozen, meaning the existence of a "glassy bead layer".

\subsection{Adsorbed Chain Beads}

The above results indicate that a "glassy polymer/bead layer" exists in the vicinity of the nanosheet surface in the strongly attractive case. Next, changing the number of adsorbed chain beads was explored as a function of the interfacial strength, and changing the number density distribution of adsorbed chain beads. The adsorbed chain
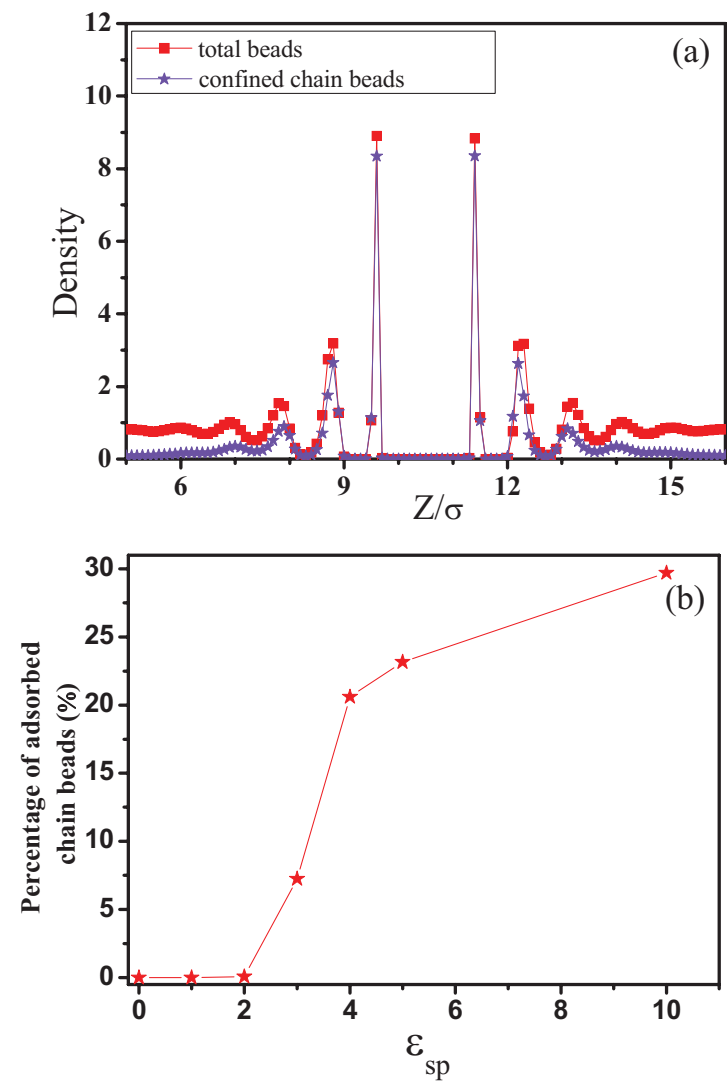

Figure 12. (a) Number density of all beads and adsorbed chain beads as a function of the distance from the nanosheet surface for strongly attractive interaction. (b) Percentage of the adsorbed chain beads vs. polymer-nanosheet interfacial interaction strength.

beads are defined as those beads whose mean squared displacement $(r(t)-r(0))^{2}$ is less than 0.05 , where $t$ is equal to $5000 \tau$. One chain is considered as "adsorbed chain" as long as one of the chain beads satisfies this requirement. The local number density distribution of the adsorbed chain beads is shown in Figure 12(a), and the total mass density profile is also shown. All beads in the first layer are strongly adsorbed chain beads. The local number density of adsorbed chain beads decreases continuously with the increase of the distance from the nanosheet surface, and drops to zero at the distance where all beads are "free". We inferred that the adsorbed bead density profile at large distance is due to the presence of long dangling tails. The percentage of adsorbed chain beads as a function of polymer-nanosheet interfacial strength is shown in Figure 12(b), which indicates that the critical interfacial interaction for the transition from "mobile layer" to "glassy layer" is between $2.0 \varepsilon$ and $3.0 \varepsilon$.

\subsection{Further Discussion}

In this work, it was found that the thickness of polymernanosheet interface is around $5 \sigma$. As mentioned above, $\sigma$ 
stands for $0.5-1 \mathrm{~nm}$, so $5 \sigma$ means $2.5-5 \mathrm{~nm}$. The thickness of the "glassy bead layer" is about $1.5 \sigma$, i.e., $0.75-1.5 \mathrm{~nm}$. Actually, Vieweg and co-workers ${ }^{[49]}$ determined the length of the immobilized modes at the filler surface and estimated the thickness of the immobilized layer to be around $1.5 \mathrm{~nm}$ using rheological data. Meanwhile, for silica filled poly(dimethylsiloxane), Arrighi and co-workers ${ }^{[50]}$ found a layer with its thickness equal to $5 \mathrm{~nm}$, whereas Litvinov and Spiess $^{[51]}$ estimated the interfacial layer to be $0.8 \mathrm{~nm}$ for their system using ${ }^{2} \mathrm{H}$ NMR as the probe. In addition, the thickness of the immobile layer varied between 0.5 and $2 \mathrm{~nm}$, while the restricted mobility layer was between 2.5 and $9 \mathrm{~nm}$, depending on the size and volume fraction of carbon black particles. ${ }^{[52-54]}$ In general, the simulated results basically agree with these experimental values. In this simulation, the maximum polymer-nanosheet interaction $\varepsilon$ is set to 10 , indicating the binding energy between polymer chains and the nanosheet is around 25 to $40 \mathrm{~kJ} \mathrm{~mol}^{-1}$. The average binding energy between poly-

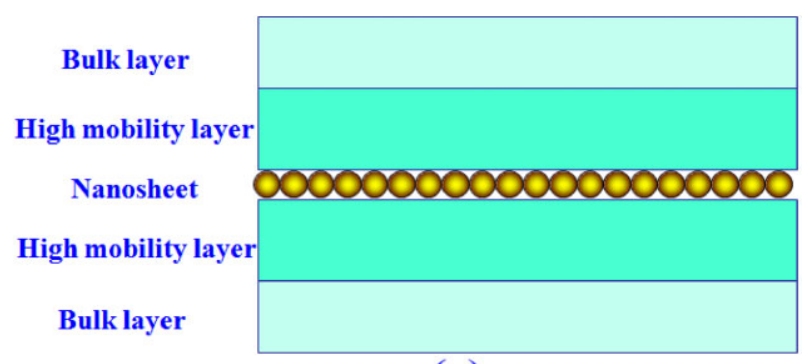

(a)

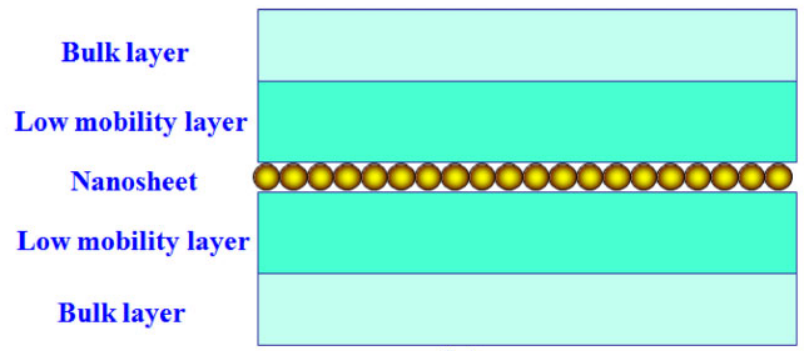

(b)

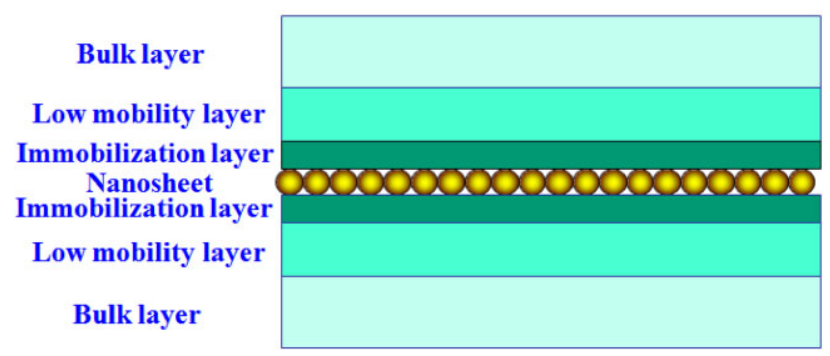

(c)

Figure 13. Proposed models for polymer chains interacting with the nanosheet. Two-layer model for (a) the purely repulsive system and (b) weakly attractive system, and (c) three-layer model for strongly attractive system. dimethylsiloxane (PDMS) chains and silica particles was reported to be $25 \mathrm{~kJ} \mathrm{~mol}^{-1}$ [55] Additionally, the binding energy between carbon black particles and styrenebutadiene polymers is around $22 \mathrm{~kJ} \mathrm{~mol}^{-1}$. [56] Hence, the simulated interfacial interaction strength is also within a realistic range according to those experimental results. In fact, as shown in Figure 10, the exchange rate became nearly zero and the polymer beads in the first layer are completely frozen at $\varepsilon_{s p}=5$.

Generally, the polymer chains span several mobility layers. Moreover, the adsorbed chains close to the nanosheet surface can undergo the adsorption/desorption processes for weak interfacial interaction, while the polymer chains completely frozen in the vicinity of the nanosheet due to strong interfacial interaction would form a "glassy polymer layer". These results agree well with experimental and theoretical studies. ${ }^{[9,42-44]}$ It is obvious that a mobility gradient of polymer beads exists near the nanosheet surface, but the "glassy layer" can only form for strongly attractive interfacial strength. Based on these results, the schemes we propose for three different interfacial strengths are shown in Figure 13. In the case of purely repulsive interaction, the polymer dynamics in the interfacial region are faster than those in the bulk state, while a slowing down of chain mobility is found for weakly attractive interaction and an "immobilized layer" appears for strongly attractive interaction.

\section{Conclusion}

By changing the polymer-nanosheet interaction, MD simulation was used to systematically investigate the static and dynamic properties of polymer melts in the presence of a nanosheet. The bead-density profiles near the nanosheet surface show different oscillation behavior for different polymer-nanosheet interfacial interactions. The bond, segment and chain orientations near the interface exhibit significant differences from that in bulk state. Meanwhile, it was observed that polymer chains are elongated and flattened along their ellipsoids close to the surface independent of the interfacial strength, which could be ascribed to the configurational entropy. The glasstransition temperature is obviously dependent on the interfacial interaction strength. It decreases for purely repulsive interaction and increases for attractive interaction, compared to the pure system. The dynamic analysis, including spatial distribution of the population of beads, interfacial beads exchange rate, desorption dynamics, translational, and orientation mobility, indicates that the "glassy polymer layer" exists for strongly attractive interfacial interaction. Actually, the results obtained here could provide some insight into polymer-nanosheet interfacial behavior.

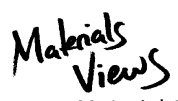

www.MaterialsViews.com 
Acknowledgements: This work was supported by the NSF of China (21274011), the National High Technology Research and Development Program of China (863 Program) (2009AA03Z338), the Foundation for Innovative Research Groups of the NSF of China (51221002), the National Basic Research Program (2011CB706900), the "Chemical Grid Project" and the Excellent Talents Funding of BUCT.

Received: July 22, 2013; Revised: August 30, 2013; Published online: October 7, 2013; DOI: 10.1002/mats.201300127

Keywords: glassy layer; interfacial gradient; molecular dynamics; nanosheet-polymer interface

[1] L. S. Schadler, S. K. Kumar, B. C. Benicewicz, S. L. Lewis, S. E. Harton, MRS Bull. 2007, 32, 335.

[2] L. S. Schadler, L. C. Brinson, W. G. Sawyer, JOM 2007, 59, 53.

[3] Z. H. Wang, J. Liu, S. Z. Wu, W. C. Wang, L. O. Zhang, Phys. Chem. Chem. Phys. 2010, 12, 3014.

[4] R. D. Priestley, C. J. Ellison, L. J. Broadbelt, J. M. Torkelson, Science 2005, 309, 456.

[5] S. H. Anastasiadis, K. Karatasos, G. Vlachos, Phys. Rev. Lett. 2000, 84, 915

[6] S. Kaufman, W. P. Slichter, D. D. Davis, J. Polym. Sci., Part A: Polym. Chem. 1970, 9, 829.

[7] J. O’Brien, E. Cashell, G. E. Wardell, V. J. McBrierty, Macromolecules 1976, 9, 653.

[8] L. T. Vo, S. H. Anastasiadis, E. P. Giannelis, Macromolecules 2011, 44, 6162.

[9] L. Chen, K. Zheng, X. Y. Tian, K. Hu, R. X. Wang, C. Liu, Y. Li, P. Cui, Macromolecules 2010, 43, 1076.

[10] C. G. Robertson, M. Rackaitis, Macromolecules 2011, 44, 1177.

[11] R. B. Bogoslovov, C. M. Roland, A. R. Ellis, A. M. Randall, C. G. Robertson, Macromolecules 2008, 41, 1289.

[12] C. G. Robertson, C. J. Lin, M. Rackaitis, C. M. Roland, Macromolecules 2008, 41, 2727.

[13] C. G. Robertson, C. M. Roland, Rubber Chem. Technol. 2008, 81, 506.

[14] J. Liu, Y. Wu, J. X. Shen, Y. Y. Gao, L. O. Zhang, D. P. Cao, Phys. Chem. Chem. Phys. 2011, 13, 13058.

[15] D. Brown, P. Mele, S. Marceau, N. D. Alberola, Macromolecules 2003, 36, 1395.

[16] D. Brown, V. Marcadon, P. Mele, N. D. Alberola, Macromolecules 2008, 41, 1499.

[17] D. Barbier, D. Brown, A. C. Grillet, S. Neyertz, Macromolecules 2004, 37, 4695.

[18] T. V. M. Ndoro, E. Voyiatzis, A. Ghanbari, D. N. Theodorou, M. C. Bohm, F. Muller-Plathe, Macromolecules 2011, 44, 2316.

[19] T. V. M. Ndoro, M. C. Bohm, F. Muller-Plathe, Macromolecules 2012, 45, 171.

[20] A. Ghanbari, T. V. M. Ndoro, F. Leroy, M. Rahimi, M. C. Bohm, F. Muller-Plathe, Macromolecules 2012, 45, 572.

[21] F. W. Starr, T. B. Schroder, S. C. Glotzer, Macromolecules 2002, $35,4481$.
[22] A. Papon, H. Montes, M. Hanafi, F. Lequeux, L. Guy, K. Saalwachter, Phys. Rev. Lett. 2012, 108, 065702.

[23] A. Karatrantos, R. J. Composto, K. I. Winey, N. Clarke, Macromolecules 2011, 44, 9830.

[24] A. Karatrantos, R. J. Composto, K. I. Winey, M. Kroger, N. Clarke, Macromolecules 2012, 45, 7274.

[25] G. N. Toepperwein, N. C. Karayiannis, R. A. Riggleman, M. Kroger, J. J. de Pablo, Macromolecules 2011, 44, 1034.

[26] G. N. Toepperwein, R. A. Riggleman, J. J. de Pablo, Macromolecules 2012, 45, 543.

[27] C. Batistakis, A. V. Lyulin, M. A. J. Michels, Macromolecules 2012, 45, 7282.

[28] K. Johnston, V. Harmandaris, Soft Matter 2012, 8, 6320.

[29] V. A. Harmandaris, K. C. Daoulas, V. G. Mavrantzas, Macromolecules 2005, 38, 5796.

[30] M. Vogel, Macromolecules 2009, 42, 9498.

[31] O. Borodin, G. D. Smith, R. Bandyopadhyaya, O. Byutner, Macromolecules 2003, 36, 7873.

[32] J. A. Torres, P. F. Nealey, J. J. de Pablo, Phys. Rev. Lett. 2000, 85, 3221.

[33] J. Liu, S. Z. Wu, L. O. Zhang, W. C. Wang, D. P. Cao, Phys. Chem. Chem. Phys. 2011, 13, 518.

[34] J. Liu, D. P. Cao, L. O. Zhang, J. Phys. Chem. C 2008, 112, 6653.

[35] J. S. Smith, O. Borodin, G. D. Smith, E. M. Kober, J. Polym. Sci., Part B: Polym. Phys. 2007, 45, 1599.

[36] J. Liu, D. P. Cao, L. O. Zhang, W. C. Wang, Macromolecules 2009, 42, 2831.

[37] S. Plimpton, J. Comput. Phys. 1995, 117, 1.

[38] K. C. Daoulas, V. A. Harmandaris, V. G. Mavrantzas, Macromolecules 2005, 38, 5780 .

[39] P. Y. Lai, J. Chem. Phys. 1995, 103, 5742.

[40] W. E. Wallace, J. H. van Zanten, W. L. Wu, Phys. Rev. E 1995, 52, R3329.

[41] J. A. Forrest, K. D. Veress, J. R. Dutcher, Phys. Rev. E 1997, 56, 5705.

[42] S. E. Harton, S. K. Kumar, H. C. Yang, T. Koga, K. Hicks, E. Lee, J. Mijovic, M. Liu, R. S. Vallery, D. W. Gidley, Macromolecules 2010, 43, 3415.

[43] S. Merabia, P. Sotta, D. R. Long, Macromolecules 2008, 41, 8252.

[44] J. Moll, S. K. Kumar, Macromolecules 2012, 45, 1131.

[45] P. V. Komarov, Y. T. Chiu, S. M. Chen, P. Reineker, Macromol. Theory Simul. 2010, 19, 64.

[46] S. K. Kumar, M. Vacatello, D. Y. Yoon, Macromolecules 1990, 23, 2189.

[47] H. Eslami, F. Muller-Plathe, J. Phys. Chem. B. 2009, 113, 5568.

[48] S. K. Kumar, M. Vacatello, D. Y. Yoon, J. Chem. Phys. 1988, 89, 5206.

[49] S. Vieweg, R. Unger, E. Hempel, E. Donth, J. Non-Cryst. Solids 1998, 235-237, 470.

[50] V. Arrighi, J. S. Higgins, A. N. Burgess, G. Floudas, Polymer 1998, 39, 6369.

[51] V. M. Litvinov, H. W. Spiess, Makromol. Chem. 1991, 192, 3005.

[52] J. O'Brien, E. Cashell, G. E. Wardell, V. J. McBrierty, Macromolecular 1976, 9, 653.

[53] D. C. Douglass, V. J. McBrierty, Polym. Eng. Sci. 1979, 19, 1054.

[54] I. Pliskin, N. J. Tokita, Appl. Polym. Sci. 1972, 16, 473.

[55] D. E. Hanson, M. Hawley, R. Houlton, K. Chitanvis, P. Rae, B. E. Orler, D. A. Wrobleski, Polymer 2005, 46, 10989.

[56] F. Bueche, Rubber Chem. Technol. 1960, 10, 107. 